\title{
EMPRESAS DE CRECIMIENTO ACELERADO Y PRODUCTIVIDAD: EVIDENCIA MICROECONÓMICA PARA CHILE*
}

\author{
Mario Canales \\ Banco Central de Chile \\ Álvaro García Marín \\ Universidad de los Andes
}

\begin{abstract}
RESUMEN: Existe abundante evidencia que muestra la significativa contribución de las empresas de crecimiento acelerado - llamadas "gacelas" por la literatura - al empleo y la actividad agregada. Sin embargo, aún existe una brecha de conocimiento con respecto a su contribución al crecimiento de la productividad. En este trabajo se aborda esta materia, utilizando para ello información administrativa
\end{abstract}

Mario Canales. Economista y magíster en análisis económico por la Universidad de Chile. Investigador del Banco Central de Chile. Email: mcanales@bcentral.cl.

Álvaro García Marín. Economista de la Universidad de Chile y PhD en administración por la UCLA. Profesor de la Universidad de los Andes. Email: agarciam@ uandes.cl.

* Los autores agradecen los comentarios y sugerencias del coeditor de Estudios Públicos, Rodrigo Wagner, de los dos árbitros anónimos de la revista, y de Roberto Álvarez, Raphael Bergoeing, Claudio Bravo-Ortega, José Luis Contreras, Fernando Greve, Rodrigo Miranda, Lucas Navarro, Joseph Ramos, Matías Tapia, Juan Marcos Wlasiuk y Andrés Zahler. Todos los resultados fueron derivados a partir de datos innominados, lo que garantiza la confidencialidad de la información tributaria. Álvaro García Marín agradece la hospitalidad del Banco Central de Chile. Este trabajo contó con financiamiento de la Comisión Nacional de Productividad de Chile. Cualquier error en el artículo es de exclusiva responsabilidad de los autores y no representa la posición del Banco Central de Chile. 
del universo de empresas formales chilenas, para el período comprendido entre 2005 y 2015 . Se concluye que las empresas de crecimiento acelerado explican una fracción desproporcionada de la creación de empleo y del crecimiento de la productividad agregada de la economía chilena. En base a los resultados, el trabajo argumenta que las políticas de fomento productivo debiesen, más que ocupar criterios de tamaño, enfocarse en crear condiciones para el surgimiento de un mayor número de este tipo de empresas.

PALABRAs Clave: tamaño de empresas, productividad, gacelas, creación de empleo.

RECIBIDO: octubre 2018; ACEPTADO: noviembre 2018.

Clasificación JEL: D22; D24; J23; L25.

\title{
HIGH-GROWTH FIRMS AND PRODUCTIVITY: MICROECONOMIC EVIDENCE FOR CHILE
}

\begin{abstract}
There is abundant evidence about the sizable contribution of high-growth firms - known as "gazelles" in the literature-, to employment and aggregate activity. However, there is still a knowledge gap regarding their contribution to productivity growth. This article studies the subject using administrative information for the universe of Chilean formal firms for the period from 2005 to 2015. It concludes that high-growth firms account for a disproportionate share of job creation and aggregate productivity growth in the Chilean economy. Based on these findings, the article argues that production development policies, rather than using size criteria, should focus on creating conditions for the emergence of a greater number of high-growth firms.
\end{abstract}

KEYWORDS: company size, productivity, gazelles, job creation.

RECEIVED: October 2018; ACCEPTED: November 2018.

JEL Classification: D22; D24; J23; L25.

\section{INTRODUCCIÓN}

\footnotetext{
E 1 debate acerca de la importancia del tamaño de las empresas para la dinámica de las economías está pronto a cumplir cuatro décadas. La evidencia provista inicialmente por Birch $(1979 ; 1981)$ sugería un rol preponderante para las empresas pequeñas como creadoras de empleo. Sin embargo, esta visión fue paulatinamente desacreditada
} 
por estudios posteriores (Davis et al. 1996b; Haltiwanger et al. 2013, entre otros). El consenso actual sugiere que, de hecho, ni las empresas pequeñas ni las grandes son las que crean la mayor parte del empleo en las economías. La creación de empleo se encuentra focalizado en un grupo pequeño de empresas que experimentan procesos de crecimiento acelerado, conocidas como gacelas (bautizadas así por Birch 1987; ver Henrekson y Johansson 2010).

Si bien la controversia sobre la relación entre tamaño de empresas y creación de empleo se encuentra relativamente zanjada, una disputa aún más importante persiste: la importancia del tamaño de las empresas para el crecimiento de la productividad. A la fecha, la evidencia acerca de la contribución relativa de las empresas pequeñas, grandes y de crecimiento acelerado al crecimiento de la productividad es escasa, y la evidencia disponible se centra en países desarrollados. ${ }^{1}$ Esta pregunta es relevante no sólo desde un punto de vista académico, sino que también desde un punto de vista de políticas públicas. La productividad en Chile, y en gran parte de países desarrollados, se ha estancado en las últimas décadas. Por tanto, entender qué tipo de empresas contribuyen en mayor medida al crecimiento de la productividad puede significar un importante impacto sobre la focalización de los programas de fomento productivo.

Este artículo estudia la relación entre productividad y tamaño de las empresas, para lo cual utiliza información administrativa del universo de empresas formales chilenas, en el período comprendido entre 2005 y 2015. Estos datos cubren todos los sectores productivos de la economía, permitiendo una mayor representatividad de los resultados que estudios previos. El caso de Chile es de interés, pues pese a experimentar altas tasas de crecimiento de la productividad en el período 1986-2000, en promedio, las empresas chilenas muestran grandes brechas de productividad en relación con los países de la Organización

${ }^{1}$ Du y Temouri (2015) muestran que, para el caso del Reino Unido, las empresas de crecimiento acelerado experimentan mejoras en productividad. Bianchini et al. (2017) y Guillamón et al. (2017) muestran que las empresas de crecimiento acelerado son más eficientes en Italia y España. En el otro extremo, Daunfeldt y Halvarsson (2015) encuentran una relación insignificante - e incluso negativa - entre crecimiento de la productividad y crecimiento acelerado en un panel de empresas suecas. 
para la Cooperación y el Desarrollo Económicos (OCDE). En efecto, evidencia reciente en Canales y García (2017) y en el Informe Anual de la Comisión Nacional de Productividad de Chile (2017) sugiere que los trabajadores de la OCDE son en promedio 1,5 veces más productivos que los trabajadores chilenos.

El trabajo documenta una serie de hechos estilizados. Primero, se estudia la contribución de los distintos tamaños de empresas al empleo, ventas y valor agregado de la economía. Los resultados muestran que, de hecho, las empresas relativamente grandes (definidas como aquellas que emplean más de 200 trabajadores) son las que contribuyen mayoritariamente al empleo y ventas de la economía. Estos resultados son consistentes con evidencia derivada en economías desarrolladas. ${ }^{2}$ Pese a representar una fracción menor del total de empresas, las grandes empresas aportan más de la mitad de los empleos, y aproximadamente 80 por ciento de las ventas de la economía chilena.

A continuación, el artículo muestra la contribución de las empresas de crecimiento acelerado (gacelas) a la productividad, empleo y ventas de la economía. Los resultados sugieren que, tal como en economías desarrolladas, las gacelas también tienen un rol sobresaliente en la economía chilena. Pese a representar tan sólo el 7 por ciento del total de empresas, contribuyen con cerca del 40 por ciento de los puestos de trabajo creados sobre una ventana de cinco años. Además, las gacelas contribuyen con aproximadamente el 40 por ciento del crecimiento de la productividad dentro de las empresas. En su conjunto, estos resultados califican y matizan la visión de las pequeñas y medianas empresas — pymes - como motores de la economía. ${ }^{3}$ Las empresas grandes, y en

${ }^{2}$ Véase Henrekson y Johansson (2010) para una revisión de la literatura, y Haltiwanger et al. (2013) para evidencia reciente.

3 Esta visión es transversal a distintos organizaciones gubernamentales y multilaterales. Por ejemplo, la OCDE (2018) destaca el rol de las pymes para el crecimiento económico, creación de empleo y el desarrollo de los países, y el Banco Mundial periódicamente desarrolla programas para facilitar el crecimiento de las pymes. En Chile, el gobierno celebró en noviembre de 2018 la "Semana de la PyME", la cual incluyó una serie de medidas de fomento para este grupo de empresas. En la inauguración de este evento, el ministro de Economía, Ramón Valente, aseguró que las pymes "son la columna vertebral, el alma, el espíritu de nuestro país", y además "el corazón de Chile y un importante motor de nuestra economía". Ver http:/www.superir.gob.cl/gobierno-da-inicio-a-la-semana-de-la-pyme-concelebracion-en-la-plaza-de-la-constitucion/. 
particular las empresas de crecimiento acelerado, son las que mueven el empleo y ventas en la economía chilena. ${ }^{4}$

Los resultados de este trabajo sugieren que la productividad agregada de la economía chilena podría ser mejorada simplemente fomentando el surgimiento de gacelas. El artículo, sin embargo, muestra de forma algo decepcionante que el surgimiento de gacelas no posee mayor correlación con ninguna variable observable que permita identificarlas antes de que éstas comiencen a desarrollarse de forma acelerada. Las gacelas se encuentran distribuidas de manera relativamente similar al resto de las empresas que componen la economía, y su orientación exportadora no difiere significativamente del resto de las empresas. Además, no son significativamente más productivas que compañías de similar tamaño antes de empezar a crecer. En consecuencia, se plantea que las empresas de crecimiento acelerado sólo podrían ser identificadas en forma ex post: su tamaño y productividad iniciales no son significativamente distintos al del resto de las empresas. Esto sugiere que políticas de fomento basadas sólo en cuestiones de tamaño, productividad actual o número de empleados no necesariamente predicen el surgimiento de gacelas, aunque no se descarta que exista alguna otra categorización de las empresas por la que se pueda fomentar el crecimiento de la productividad agregada. ${ }^{5}$

Una potencial fuente de preocupación relativa a los resultados del artículo radica en la identificación del rápido crecimiento de las gacelas. Evidencia para distintos países sugiere que los episodios de crecimiento acelerado son más bien esporádicos y de vida corta (Goswami et al.

\footnotetext{
${ }^{4}$ La metodología empleada no permite hacer juicios respecto a la dirección de causalidad del efecto, por lo cual el resultado sólo debe ser interpretado como una descomposición del crecimiento de la productividad. Evidencia para Reino Unido y España (Du y Temouri 2015 y Guillamón et al. 2017) muestra la existencia de un círculo virtuoso entre crecimiento de la productividad y experiencias de crecimiento acelerado. Esto sugiere que algún tercer factor (como inversión en tecnología, activos intangibles o mejoras en la administración) podría ser el causante tanto del aumento de tamaño como de las mejoras de la productividad.

${ }^{5}$ Una dimensión en la cual las empresas de crecimiento acelerado parecen ser diferentes es su edad: las gacelas tienden a ser más jóvenes que el resto de las empresas de la industria (ver proyecto Dynemp de la OECD, y Criscuolo et al. 2014, para evidencia comparativa de 18 países). Desafortunadamente, los datos con los cuales se trabaja en este artículo no incluyen información de la edad de las empresas.
} 
2018). Además, una fracción de los episodios de crecimiento acelerado pareciera responder a rebotes luego de shocks negativos, fenómeno denominado Ashenfelter's dip (ver evidencia para Suecia en Daunfeldt y Halvarsson 2015). En otros casos, el surgimiento de alto crecimiento pareciera seguir a episodios de alto crecimiento (Du y Temouri 2015; Guillamón et al. 2017). Al respecto, quisiéramos hacer notar que el punto que se desea destacar en el artículo es que estos episodios de crecimiento acelerado están acompañados por mejoras significativas en la eficiencia productiva de las empresas. Este punto no es trivial, pues podría ser que las empresas incluso se volviesen menos eficientes al aumentar su escala si es que alguna fricción les impide ajustar el uso de recursos al interior de la empresa. La principal contribución de este artículo radica, por lo tanto, en documentar las ganancias de productividad experimentadas por las empresas de rápido crecimiento. Entender los mecanismos por medio de los cuales las gacelas mejoran su productividad o la dirección de causalidad del efecto, si bien es importante, va más allá de los alcances de este trabajo. ${ }^{6}$

El resto del artículo se organiza de la siguiente forma: La sección 2 discute el marco empírico y se presenta la medida de productividad total de factores usada en las inmediatas secciones. La sección 3 presenta los datos utilizados en el trabajo. La sección 4 presenta los resultados empíricos. Finalmente, la 5 presenta las principales conclusiones.

\section{ESTIMACIÓN DE LA PRODUCTIVIDAD}

La productividad - una medida de la eficiencia con la que las unidades productivas convierten insumos en productos o bienes finalestípicamente se mide como el residuo entre producto y la contribución de los insumos utilizados por las unidades productivas. Idealmente, tanto insumos como productos deberían ser medidos en términos de unida-

${ }^{6}$ Goswami et al. (2018) discuten elementos que podrían explicar el rápido crecimiento de las gacelas. En particular, estos autores muestran que la probabilidad de experimentar un episodio de crecimiento acelerado aumenta significativamente con la actividad innovativa de las empresas. Además, empresas con más encadenamientos productivos y financieros con otras empresas domésticas y multinacionales, y que pagan salarios más altos experimentan con mayor frecuencia episodios de rápido crecimiento. 
des físicas. Sin embargo, en la práctica dicha información no se encuentra disponible. En consecuencia, gran parte de los investigadores optan por aproximar insumos y productos en términos de valores monetarios: gasto en insumos y valor de las ventas, respectivamente. La medida de productividad multifactorial resultante se conoce como productividad total de factores basada en ventas (TFPR, por sus siglas en inglés), para diferenciarla del real sujeto de interés, donde insumos y productos se miden en términos de unidades físicas, conocida como productividad total de factores basada en cantidades (TFPQ, por sus siglas en inglés).

Para estimar la productividad, se especifica una función de producción Cobb-Douglas para cada rama industrial $s$, con trabajo $(L)$, capital $(K)$ y materiales $(M)$ como insumos productivos:

$$
q_{i t}=\alpha_{l}^{S} l_{i t}+\alpha_{k}^{S} k_{i t}+\alpha_{m}^{s} m_{i t}+\omega_{i t}+\varepsilon_{i t}
$$

Donde todas las variables en minúscula están expresadas en logaritmos; $q_{i t}$ representa las ventas de la unidad productiva $i$ en el año $t ; l_{i t}$ representa trabajo; $k_{i t}$, capital; $m_{i t}$, materiales; $\omega_{i t}$ es la medida de productividad, mientras que $\varepsilon_{i t}$ es un término de error que no se encuentra correlacionado con el nivel de los insumos productivos.

Idealmente, insumos y productos deberían ser deflactados con índices de precios específicos por planta industrial. ${ }^{7}$ Sin embargo, la base de datos sólo proporciona información de producción en términos de ventas, por lo que las metodologías usuales de estimación únicamente permitirían recuperar medidas de productividad basada en ventas (TFPR, como ya vimos).

Debido a que no existe información de unidades físicas en la base de datos del Servicio de Impuestos Internos de Chile (SII), se opta por aplicar la metodología impulsada por De Loecker (2011). Este autor modifica la metodología propuesta por Ackerberg et al. (2015), aproximando el componente de precios con una forma funcional particular

${ }^{7}$ Foster et al. (2008) muestran que la eficiencia de las empresas en términos de TFPQ se relaciona negativamente con los precios. Esto implica que TFPR subestima las ganancias de eficiencia de las empresas. Una literatura reciente muestra que utilizar TFPR en lugar de TFPQ no es inocuo. García y Voigtländer (por aparecer) muestran que utilizar medidas de eficiencia basadas en unidades físicas es clave para encontrar ganancias de eficiencia asociadas a la entrada a los mercados de exportación. 
para la demanda de los productos. En particular, se considera un sistema de demanda estándar de diferenciación horizontal con elasticidad de sustitución constante (CES) del siguiente tipo para cada sector industrial $s:{ }^{8}$

$$
Q_{i t}=Q_{s t}\left(P_{i t} / P_{s t}\right)^{\eta_{s}}
$$

El sistema de demanda (2) implica que la demanda por el producto de las empresas depende de su propio precio $\left(P_{i t}\right)$, del precio promedio del sector $\left(P_{s t}\right)$ y de la demanda agregada $\left(Q_{s t}\right) .{ }^{9}$ Según muestran Klette y Griliches (1996) como también Levinsohn y Melitz (2006), la demanda (2) puede ser usada para controlar el componente de precios $P_{i t}$ en la estimación de la función de producción (1) cuando no existe información de unidades físicas del producto. En efecto, utilizando (2), las ventas de una empresa pueden ser escritas en función de unidades físicas y variables agregadas:

$$
R_{i t}=P_{i t} \cdot Q_{i t}=Q_{i t}^{\left(\eta_{s}+1\right) / \eta_{s}} Q_{s t}^{-1 / \eta_{s}} P_{s t}
$$

Una vez que se aplican los deflactores industriales, la ecuación a estimar para la función de producción en términos de ventas queda de la siguiente manera:

$$
\underline{r}_{i t}=\beta_{l}^{s} l_{i t}+\beta_{k}^{s} k_{i t}+\beta_{m}^{s} m_{i t}+\beta_{q}^{s} q_{s t}+\omega_{i t}^{*}+\varepsilon_{i t}
$$

${ }^{8}$ La metodología original de De Loecker (2011) incluye además un shock de demanda no observado en la especificación de demanda, que posteriormente el autor identifica explotando diferencias en el nivel de protección aplicada a una muestra de productores textiles. A diferencia de De Loecker (2011), en este artículo no incluimos el shock de demanda en la especificación de demanda, pues la estimación comprehensiva de productividad, para el universo de empresas en la economía, hace poco factible la construcción de instrumentos para cada subsector industrial como en De Loecker (2011). Esto implica que la medida de productividad podría potencialmente reflejar innovaciones de demanda a nivel de unidades productivas. Sin embargo, la medida de productividad sí capturará shocks de demanda a nivel de industrias, que se reflejen en cambios en Qst.

${ }^{9}$ Este sistema de demanda, junto con el supuesto de competencia monopolística, implica que para las empresas resulta óptimo cobrar un precio igual a un markup constante $\mathrm{s} /(\mathrm{s}+1)$ sobre el costo marginal. 
Donde $\underline{r}_{i t}$ corresponde a ventas deflactadas con índices de precios industriales. Como se puede ver, las elasticidades de insumo-producto de la función de producción basada en ventas combinan parámetros de oferta $\left(a_{h}^{s}\right.$, donde $\left.h \in\{l, k, m\}\right)$ y demanda $\left(n_{s}\right)$. De hecho, es posible mostrar que las elasticidades de la forma reducida (4) se relacionan con las verdaderas elasticidades $a_{h}$ de acuerdo a la fórmula $\beta_{h}^{S}=\left[\left(\eta_{s}+1\right) / \eta_{s}\right] \alpha_{h}^{S}$, para $h \in\{l, k, m\}$. El valor del parámetro $n_{s}$, en tanto, puede ser recuperado directamente del valor de $\beta_{q}^{S}=1 /\left|\eta_{s}\right|$ Finalmente, la productividad total de factores en términos de unidades físicas puede ser computada de acuerdo a la expresión (5):

$$
\omega_{i t}=\omega_{i t}^{*}\left(\frac{\eta_{s}}{\eta_{s}+1}\right)=\left(\underline{r}_{i t}-\beta_{l}^{s} l_{i t}-\beta_{k}^{s} k_{i t}-\beta_{m}^{s} m_{i t}-\beta_{q}^{s} q_{s t}\right)\left(\frac{\eta_{s}}{\eta_{s}+1}\right)
$$

Para estimar (4), se sigue la metodología propuesta por Ackerberg et al. (2015), quienes extienden el trabajo de Olley y Pakes (1996) y de Levinsohn y Petrin (2003). Un supuesto crucial para estimar la función de producción es que la productividad de las empresas sigue un proceso markoviano de primer orden: $\omega_{i t}=g\left(\omega_{i t-1}\right)+\xi_{i t}$. Este supuesto implica que las innovaciones $\xi_{i t}$ a la productividad no se correlacionan con la información disponible en el período t.

El punto de partida de Ackerberg et al. (2015) —al igual que LPes asumir una demanda de materiales del tipo:

$$
m_{i t}=g_{t}\left(k_{i t}, l_{i t}, \omega_{i t}\right)
$$

Ésta permite expresar la productividad como una función de los insumos productivos, asumiendo invertibilidad de la función en $m_{i t}($. en $w_{i t}$ :

$$
\omega_{i t}=g_{t}^{-1}\left(m_{i t}, l_{i t}, k_{i t}\right) \equiv h_{t}\left(m_{i t}, l_{i t}, k_{i t}\right)
$$

Donde $g_{t}^{-1}\left(m_{i t}, l_{i t}, k_{i t}\right)$ corresponde a la función inversa de la función $g_{t}(\cdot)$. En la primera etapa de la rutina de Ackerberg et al. (2015), se computa un estimador consistente del producto esperado (ecuación 4), aproximando productividad con la expresión derivada en 
(7). ${ }^{10} \mathrm{El}$ producto esperado — definido $\phi_{t}$ de aquí en adelante- excluye el componente $\varepsilon_{i t}$, y corresponde a la siguiente expresión:

$$
\phi_{t}(\cdot)=\beta_{l}^{s} l_{i t}+\beta_{k}^{s} k_{i t}+\beta_{m}^{s} m_{i t}+\beta_{q}^{s} q_{s t}+h_{t}\left(m_{i t}, l_{i t}, k_{i t}\right)
$$

Debido a que la función $h_{t}(\cdot)$ es una función de trabajo, materiales y stock de capital, los coeficientes $\beta^{S} \equiv\left\{\beta_{l}^{S}, \beta_{k}^{S}, \beta_{m}^{S}\right\}$ no pueden ser identificados en la primera etapa. Sin embargo, bajo el supuesto de que la productividad sigue un proceso markoviano de primer orden (ver Olley y Pakes 1996), las innovaciones a la productividad debieran estar no correlacionadas con valores pasados de los insumos productivos. Pese a que la productividad no es observada, dado un estimador de mínimos cuadrados ordinarios (MCO) de producto esperado, es posible computar la productividad de las empresas para cada potencial vector de coeficientes $\tilde{\beta}^{S}$ :

$$
\omega_{i t}\left(\widetilde{\beta}^{s}\right)=\hat{\phi}_{i t}-\left(\widetilde{\beta_{l}^{S}} l_{i t}+\widetilde{\beta_{k}^{s}} k_{i t}+\widetilde{\beta_{m}^{s}} m_{i t}+\widetilde{\beta_{q}^{S}} q_{s t}\right)
$$

Finalmente, para cada candidato $\tilde{\beta}^{s}$, se computa la innovación al proceso de productividad, $\xi_{i t+1}\left(\tilde{\beta}^{s}\right) \equiv \omega_{i t+1}\left(\tilde{\beta}^{s}\right)-\omega_{i t}\left(\tilde{\beta}^{s}\right)$, y los parámetros se estiman por Método Generalizado de Momentos (MGM) bajo el supuesto de que la innovación de productividad no se correlaciona ni con los insumos estáticos rezagados (materiales y trabajo), ni con el capital contemporáneo, ni con el producto agregado del sector rezagado:

$$
E\left\{\xi_{i t+1}\left(\beta_{m}, \beta_{k}, \beta_{l}, \beta_{q}\right)\left(m_{i t} l_{i t} k_{i t+1} q_{s t}\right)\right\}=0
$$

\section{DATOS}

El trabajo utiliza información administrativa innominada para un panel de empresas durante el período 2005-2015. La información es provista por el SII y cubre el universo de empresas formales. Éstas son

${ }^{10}$ Las funciones $g_{t}$ y $h_{t}$ son desconocidas. Siguiendo al resto de la literatura de estimación de productividad, aproximamos esta función utilizando polinomios flexibles en todos los argumentos de la función. Las estimaciones presentadas en este artículo utilizan polinomios de segundo orden. 
identificadas por medio de un RUT Ficticio (RF) asociado a su Rol Único Tributario (RUT), los cuales son asignados por el SII a todas las personas jurídicas que inician formalmente sus actividades. El RUT es personal e intransferible, por lo que sigue al titular del RUT durante toda su existencia legal, y se mantiene inalterado incluso si la unidad productiva cambia de ubicación geográfica. ${ }^{11}$

La totalidad de la información utilizada para caracterizar a las empresas chilenas proviene de los formularios 22, 29 y 1887 del SII. El formulario 22 ("Declaración anual de rentas") proporciona información de ventas, activos fijos, insumos intermedios y masa salarial. El formulario 1887 ("Declaración jurada anual sobre rentas") proporciona información sobre el número de trabajadores en cada empresa. Finalmente, el formulario 29 ("Declaración mensual y pago simultáneo de impuestos") es utilizado para determinar el estatus exportador.

Para el período 2005-2015, en promedio, existen aproximadamente 500 mil empresas que reportan ventas positivas en el formulario 22. Sin embargo, de este total sólo el 80 por ciento reporta insumos intermedios, y 50 por ciento reporta activos fijos. Adicionalmente, al cruzar las bases de datos de empresas (formulario 22), con las de trabajadores (formulario 1887), únicamente fue posible determinar empleo positivo en el 50 por ciento de los RUT. Consecuentemente, la muestra final considera sólo 180 mil empresas en promedio por año, que corresponden al subconjunto de empresas en las que: (i) todas las variables de interés (ventas, empleo, insumos intermedios y activos fijos) tienen valores estrictamente positivos, y (ii) la distribución de ventas por trabajador no muestra valores poco realistas (se recorta el 1 por ciento superior e inferior de la distribución de ventas por trabajador). La muestra representa el 71 por ciento del empleo asalariado, y el 82 por ciento del

${ }^{11}$ Un potencial problema de utilizar identificadores tributarios radica en que estos identifican, como distintas empresas, a unidades que cambian de rol tributario en años consecutivos. Para evitar este problema, se realiza un análisis de predecesores-sucesores, utilizando información de los trabajadores empleados en cada unidad productiva. La información sobre la nómina de trabajadores se obtiene de las declaraciones de renta de los trabajadores, las cuales especifican las empresas (RUT) para las que trabajan. En todos aquellos casos en que más del 80 por ciento migran desde un RUT a otro distinto en años consecutivos, se considera a ambos RUT como una única empresa. Este ajuste permite identificar a aproximadamente 1.500 empresas que cambian de RUT. 
valor bruto de producción de la economía, pese a que en términos de número de empresas sólo representa un tercio de los RUT con ventas positivas. ${ }^{12}$ Esto sugiere que gran parte de los RUT sin información de activos fijos y empleados corresponde a empresas con baja actividad productiva real. En términos de composición industrial, un poco más de la mitad de las empresas de la muestra se concentran en el sector terciario de la economía: servicios. En contraste, manufacturas y construcción contribuyen cada uno con aproximadamente el 10 por ciento de las empresas, mientras que los sectores silvo-agropecuario y pesca, y transportes y comunicaciones aportan con otro 7 por ciento de las empresas. Finalmente, minería y electricidad, gas y agua son los sectores más pequeños, representando en conjunto apenas 1 por ciento de las empresas chilenas. ${ }^{13}$

\section{RESULTADOS}

En esta sección se muestran los principales resultados del trabajo. La sección comienza presentando las definiciones de tamaño con las cuales se trabaja a lo largo del artículo. Luego, se discute la contribución de los distintos tamaños de empresas al crecimiento de las ventas y creación de empleo agregado. Finalmente, la sección concluye estudiando la contribución de los distintos tipos de empresas a la productividad agregada.

\subsection{Caracterización de empresas por tamaño}

Antes de proceder a la caracterización de las empresas por tamaño, se definen las categorías que se utilizarán a lo largo del documento. Tradicionalmente, tanto la literatura académica como las instituciones

12 Ambas cifras se refieren al año 2013 (última actualización de las cuentas nacionales), lo cual permite comparar la suma de las ventas de las empresas directamente con el valor bruto de producción computado en la construcción de las tablas de insumo producto. Para determinar la representatividad de la muestra en términos de empleo y producción, se compara la información de la base de datos utilizada en este artículo con información oficial de la Encuesta Nacional de Empleo (ENE) y de las Cuentas Nacionales del Banco Central de Chile.

${ }^{13}$ Para aminorar la influencia de datos extremos en los resultados, se excluyen todas las observaciones en las cuales las ventas por trabajador son mayores que el percentil 99, o menores al percentil 1 de la distribución de ventas por trabajador. 
de fomento productivo definen el tamaño en términos de dos variables: empleo y ventas. Por ejemplo, los programas de la Corporación de Fomento de la Producción (Corfo) utilizan criterios de ventas a fin de determinar el punto de corte para postular a sus programas de emprendimiento, mientras que la Organización para la Cooperación y el Desarrollo Económicos (OCDE) define el tamaño de empresas en términos del número de empleados. Consecuentemente, las dos clasificaciones de tamaño consideradas en el artículo — resumidas en el cuadro 1- se basan en el nivel de ventas y empleo de las empresas para definir los umbrales que definen a cada categoría.

Cuadro 1. CLASIFICACIONES DE TAMAÑO POR VENTAS Y EMPLEO

\begin{tabular}{lll}
\hline & Empleo & Ventas \\
\hline Pequeña & $1-49$ trabajadores & $2.400-25.000$ UF \\
Mediana & $50-199$ trabajadores & $25.000-100.000$ UF \\
Grande & +200 trabajadores & +100.000 UF \\
\hline
\end{tabular}

La clasificación de tamaño en términos de empleo se define en la segunda columna del cuadro 1. Las empresas con empleo menor a 50 trabajadores son definidas como micro o pequeñas empresas. Las empresas medianas emplean entre 50 y 199 trabajadores y las grandes, sobre 200 trabajadores. La clasificación de tamaño en términos de ventas (columna 3) sigue la clasificación de la Corfo, donde se expresan las ventas anuales en unidades de fomento (UF). Las empresas con ventas menores a 2.400 UF (aproximadamente 100 mil dólares) son clasificadas como microempresas. Las pequeñas empresas son aquellas con ventas entre 2.400 y 25.000 UF (aproximadamente un millón de dólares). Una vez que las empresas sobrepasan las 25.000 UF de ventas anuales son clasificadas como medianas, y si exceden las 100.000 UF (cuatro millones de dólares) son catalogadas como grandes.

El cuadro 2 compara las clasificaciones de tamaño por ventas y empleo en cuanto a la confluencia de criterios: cuántas veces las empresas son clasificadas bajo el mismo tamaño en ambas clasificaciones simultáneamente. Como se puede observar, pese a que el mayor número de observaciones se encuentran en la diagonal de la matriz - lo cual implica concordancia en los criterios de tamaño-, existe una importante heterogeneidad. Por ejemplo, aproximadamente $15 \mathrm{mil}$ 
empresas son clasificadas como pequeñas en términos de empleo, pero como medianas o grandes en términos de ventas, es decir, tienen ventas que sobrepasan el millón de dólares anuales. Aún más, el 40 por ciento de las empresas medianas en términos de empleo tienen ventas mayores a 5 millones de dólares. Esto nos lleva al primer resultado de este trabajo.

Cuadro 2. DISTRIBUCIÓN DE EMPRESAS EN TÉRMINOS DE EMPLEO Y VENTAS (PROMEDIO 2005-15)

\begin{tabular}{lcccc}
\hline & $\begin{array}{c}\text { Pequeña } \\
(1-49 \text { empl. })\end{array}$ & $\begin{array}{c}\text { Mediana } \\
(50-199 \text { empl. })\end{array}$ & $\begin{array}{c}\text { Grande } \\
(>200 \text { empl. })\end{array}$ & Total \\
\hline $\begin{array}{l}\text { Pequeña (<25.000 UF } \\
\text { de ventas) }\end{array}$ & 153.564 & 1.143 & 17 & 154.724 \\
$\begin{array}{l}\text { Mediana (25.000- } \\
100.000 \text { UF de ventas) }\end{array}$ & 13.168 & 3.345 & 304 & 16.817 \\
$\begin{array}{l}\text { Grande (>=100.000 UF } \\
\text { de ventas) }\end{array}$ & 2.473 & 3.131 & 2.185 & 7.789 \\
\hline Total & 169.205 & 7.619 & 2.506 & 179.330 \\
\hline
\end{tabular}

Nota: El cuadro muestra la distribución de empresas por tamaño en términos de ventas (hacia abajo) y de empleo (hacia la derecha). Cada celda muestra el número promedio de empresas en cada categoria de tamaño.

La imperfecta correlación entre las clasificaciones de tamaño por empleo y ventas es relevante, pues sugiere que existe una gran heterogeneidad en el tipo de empresas dentro de cada categoría de tamaño. Para ver esto, nótese que a medida que nos desplazamos desde el extremo superior-derecho (alto número de trabajadores y bajos niveles de ventas) al inferior-izquierdo (bajo número de trabajadores y altos niveles de ventas) del cuadro 2, la productividad laboral de las empresas - medida como ventas por trabajador - aumenta. El cuadro 3 confirma este resultado, mostrando el nivel promedio de ventas por trabajador al interior de cada categoría de tamaño. Por ejemplo, las ventas por trabajador dentro de las empresas pequeñas en términos de empleo varían desde las 969 UF anuales (50 mil dólares) a 7.777 UF anuales (325 mil dólares aproximadamente).

La alta heterogeneidad en términos de productividad laboral dentro de las clasificaciones de tamaño tradicionalmente utilizadas refuerza la noción de que las pequeñas y medianas empresas — pymes - es un 
Cuadro 3. DISTRIBUCIÓN DE EMPRESAS EN TÉRMINOS DE EMPLEO Y VENTAS

\begin{tabular}{|c|c|c|c|c|}
\hline & $\begin{array}{l}\text { Pequeña } \\
\text { (1-49 empl.) }\end{array}$ & $\begin{array}{c}\text { Mediana } \\
\text { (50-199 empl.) }\end{array}$ & $\begin{array}{c}\text { Grande } \\
\text { (>200 empl.) }\end{array}$ & $\begin{array}{l}\text { Todos los } \\
\text { tamaños }\end{array}$ \\
\hline \multicolumn{5}{|c|}{ 1. Ventas por trabajador (en UF, promedio 2005-2014) } \\
\hline $\begin{array}{l}\text { Pequeña (<25.000 UF de } \\
\text { ventas) }\end{array}$ & 969 & 212 & 58 & 889 \\
\hline $\begin{array}{l}\text { Mediana (25.000-100.000 } \\
\text { UF de ventas) }\end{array}$ & 2.459 & 658 & 211 & 1.297 \\
\hline $\begin{array}{l}\text { Grande ( }>=100.000 \text { UF de } \\
\text { ventas) }\end{array}$ & 7.777 & 3.367 & 2.670 & 2.950 \\
\hline Todos los tamaños & 1.717 & 1.891 & 2.526 & 2.148 \\
\hline \multicolumn{5}{|c|}{ 2. Trabajadores por empresa (promedio 2005-2014) } \\
\hline $\begin{array}{l}\text { Pequeña (<25.000 UF de } \\
\text { ventas) }\end{array}$ & 5,0 & 73,1 & 294,9 & 5,5 \\
\hline $\begin{array}{l}\text { Mediana ( } 25.000-100.000 \\
\text { UF de ventas) }\end{array}$ & 18,6 & 85,2 & 310,6 & 37,1 \\
\hline $\begin{array}{l}\text { Grande ( }>=100.000 \text { UF de } \\
\text { ventas) }\end{array}$ & 25,8 & 106,3 & 731,1 & 256,0 \\
\hline Todos los tamaños & 6,3 & 92,0 & 677,1 & 19,3 \\
\hline
\end{tabular}

Nota: El cuadro muestra las ventas por trabajador promedio, y el empleo de las empresas, agrupadas por categorias de tamaño en términos de ventas (hacia abajo) y empleo (hacia la derecha). Cada celda muestra el número promedio de empresas en cada categoría de tamaño.

concepto poco preciso para focalizar los recursos de los programas de fomento productivo. En efecto, si el objetivo de política busca asignar recursos a las entidades relativamente más productivas, entonces asignar subsidios de acuerdo al tamaño de las empresas no necesariamente cumpliría dicho fin, ya que al interior de cada categoría de tamaño coexisten empresas con bajos niveles de productividad por trabajador $\mathrm{y}$ otras con altos niveles de ventas por trabajador. ${ }^{14} \mathrm{Si}$ la productividad

${ }^{14}$ Existe la posibilidad de que una parte importante de las diferencias en productividad laboral al interior de tamaños en términos de empleo y ventas sea por diferencias en composición industrial. Por ejemplo, el sector de comercio, restaurantes y hoteles está compuesto de empresas más pequeñas que aquellas que pertenecen a manufacturas. Por lo tanto, es de relevancia entender cuánto de los patrones derivados en el cuadro 2 se repiten dentro de sectores. Los resultados de este ejercicio - no reportados por razones de flujo y espacio - sugieren que los patrones derivados en esta sección también se presentan dentro de sectores, aunque las brechas de productividad laboral al interior de las categorías de tamaño muestran una menor dispersión que a nivel agregado. 
es persistente, de tal forma que el nivel actual de productividad es un predictor de su valor futuro, entonces al asignar recursos a todas las pymes por igual no necesariamente llevaría a cambios significativos en la productividad agregada. Por el contrario, en un mundo ideal, los recursos de los programas de fomento debieran ser asignados a empresas con alto potencial de crecimiento de su productividad. Este tema será abordado en la sección 4.2. ${ }^{15}$

A menudo se argumenta que las empresas pequeñas debiesen ser beneficiarias de recursos públicos por el hecho de movilizar la mayor parte del empleo de las economías. El cuadro 4 investiga esta afirmación mostrando la contribución al empleo, ventas y valor agregado por tamaño de empresa. El panel superior muestra resultados utilizando tamaño por ventas, mientras que el panel inferior en términos de empleo. Como se puede apreciar, independiente de la clasificación de tamaño (por ventas o empleo), las pequeñas empresas representan el grueso de las unidades productivas en Chile: mientras 86 por ciento de las empresas son pequeñas en términos de ventas, casi el 95 por ciento del total lo son en términos de empleo (columna 2). Sin embargo, como muestran las columnas 3, 5 y 7, las empresas pequeñas sólo contribuyen con una pequeña fracción - relativo a su gran número- del empleo, ventas y valor agregado de la economía, respectivamente.

En el otro extremo, las empresas grandes aparecen como los mayores contribuyentes al empleo, ventas y valor agregado de la economía chilena. En promedio, cuando se define tamaño en términos de ventas (panel superior), las empresas grandes contribuyen con el 58 por ciento del empleo, y aproximadamente tres cuartos de las ventas y valor agregado de la economía. Al considerar clasificaciones de tamaño por empleo los resultados se suavizan, pero las empresas grandes siguen siendo el grupo con mayor preponderancia en la economía.

Podría argumentarse que la relevancia de las empresas pequeñas radica en su contribución al crecimiento - y no al nivel- de la economía. Las nuevas empresas a menudo son pequeñas, por lo que sería posible que éstas expliquen una alta fracción del crecimiento de las variables, aun cuando sólo contribuyesen con una fracción menor a la

${ }^{15}$ Una limitación de esta discusión es que está basada en productividad laboral — medida en términos de ventas por trabajador - y, por tanto, puede reflejar diferencias en capital productivo o insumos intermedios. 
Cuadro 4. CONTRIBUCIÓN A EMPLEO, VENTAS Y VALOR AGREGADO POR TAMAÑO DE EMPRESA (2005-2015)

\begin{tabular}{|c|c|c|c|c|c|c|c|c|}
\hline & (1) & (2) & (3) & (4) & (5) & (6) & (7) & (8) \\
\hline & \multicolumn{2}{|c|}{ Empresas } & \multicolumn{2}{|c|}{ Empleo } & \multicolumn{2}{|c|}{ Ventas } & \multicolumn{2}{|c|}{ Valor agregado } \\
\hline & $\mathrm{N}^{\circ}$ & $\%$ & Nivel & $\Delta 2005-15$ & Nivel & $\Delta 2005-15$ & Nivel & $\Delta 2005-15$ \\
\hline \multicolumn{9}{|c|}{ Tamaño según ventas: } \\
\hline $\begin{array}{l}\text { Pequeña } \\
\text { («25 mil UF) }\end{array}$ & 154.741 & 86,3 & 849.120 & 138.321 & 17.654 & 6.764 & 6.659 & 1.896 \\
\hline $\begin{array}{l}\text { Mediana (25 } \\
\text { mil-100 mil UF) }\end{array}$ & 16.820 & 9,4 & 624.924 & 165.432 & 18.903 & 7.224 & 7.089 & 2.404 \\
\hline $\begin{array}{l}\text { Grande } \\
(>=100 \text { mil UF) }\end{array}$ & 7.799 & 4,3 & 1.995 .964 & 968.409 & 135.260 & 73.998 & 42.747 & 17.816 \\
\hline \multicolumn{9}{|c|}{ Tamaño según empleo: } \\
\hline $\begin{array}{l}\text { Pequeña } \\
\text { (1-49 empl,) }\end{array}$ & 169.234 & 94,3 & 1.071 .787 & 320.667 & 42.520 & 18.707 & 14.094 & 5.475 \\
\hline $\begin{array}{l}\text { Mediana } \\
\text { (50-199 empl,) }\end{array}$ & 7.620 & 4,2 & 701.285 & 187.169 & 30.671 & 11.315 & 10.691 & 3.159 \\
\hline $\begin{array}{l}\text { Grande } \\
\text { (>200 empl,) }\end{array}$ & 2.506 & 1,4 & 1.696 .936 & 764.326 & 98.626 & 57.963 & 31.709 & 13.483 \\
\hline Total & 179.360 & 100,0 & 3.470 .008 & 1.272 .162 & 171.817 & 87.985 & 56.494 & 22.117 \\
\hline
\end{tabular}

Nota: El cuadro muestra la contribución de las categorias de tamaño definidas en el cuadro 1 en términos de número (columnas 1-2), empleo (columnas 3-4), ventas (columnas 5-6) y valor agregado (columnas 7-8). Valor agregado y ventas se expresan en miles de millones de pesos de 2013 .

economía agregada. Las columnas 4,6 , y 8 del cuadro 4 computan el cambio en empleo, ventas y valor agregado para cada tamaño de empresas en el período 2005 y 2015. Como se puede observar en el panel superior, las empresas pequeñas sólo contribuyen con una pequeña proporción del empleo creado en el período. Del total de 1,2 millones de empleos creados en términos netos, las empresas pequeñas sólo contribuyeron con 140 mil trabajos, apenas el 11 por ciento del total. En términos de ventas y valor agregado, el resultado es aún más impresionante, pues las empresas pequeñas sólo aportan el 8 por ciento de las ventas totales, y un poco menos del 9 por ciento del valor agregado de la economía. El panel inferior muestra que estos resultados se mantienen cualitativamente cuando se considera la clasificación de tamaño en términos de empleo. Esta discusión se formaliza en el siguiente resultado: 
Resultado 1:

Las empresas grandes aportaron la mayor parte del nivel y crecimiento del empleo, ventas y valor agregado de la economía chilena en el período 2005-2015. En contraste, pese a ser mayoría en la economía - sobre 90 por ciento del total de unidades productivas-, las empresas pequeñas y medianas sólo representan una proporción menor del empleo, ventas y valor agregado relativo a su número.

El resultado 1 conduce a cuestionar la noción —arraigada en la discusión política, e incluso académica- de que las empresas pequeñas son los mayores contribuyentes de las economías. De hecho, los resultados sugieren que este grupo es el menos importante en términos de empleo y producto. Una razón que podría explicar la baja relevancia de estas empresas podría estar relacionada con la alta tasa de fracasos de los nuevos emprendimientos. Debido a la existencia de fricciones, una gran parte de las empresas nacen pequeñas - independientemente de su productividad-, y gradualmente convergen a su tamaño óptimo, el cual debería ser mayor mientras más eficiente sean las empresas. ${ }^{16}$ Por lo tanto, las empresas menos eficientes naturalmente se mantienen pequeñas. Lo anterior conduce a subestimar la importancia de las empresas pequeñas en términos agregados, pues una porción de ellas podría experimentar procesos de crecimiento acelerado, que no serían reflejados en el cuadro 1. Para determinar la importancia de las empresas pequeñas en la economía, se hace necesario hacer seguimiento a aquéllas exitosas que escalan en períodos cortos. En la siguiente sección se aborda esta discusión, introduciendo clasificaciones dinámicas de tamaño, que intentan entender si la baja contribución de las empresas pequeñas se podría deber al hecho que excluyen a empresas de crecimiento acelerado.

${ }^{16}$ Una extensa literatura sugiere que la combinación de costos de ajuste, fricciones financieras e información incompleta previene que las empresas alcancen su tamaño óptimo al momento de su creación (ver Hsieh y Klenow 2009, 2014; Restuccia y Rogerson 2013; 2017; Asker et al. 2014). Además, una parte importante del crecimiento de las empresas parece responder a crecimiento de la base de demanda de las empresas (Foster et al. 2016; Eslava y Haltiwanger 2018). 


\subsection{Tamaño de empresas: contribución a empleo y ventas}

Una literatura reciente muestra evidencia sugiriendo que, en países desarrollados, una pequeña fracción de empresas de alto crecimiento genera la mayor parte de la creación neta de empleos. ${ }^{17}$ Este tipo de empresas - bautizadas por Birch (1987) como gacelas- serían los agentes más dinámicos en las economías.

No hay acuerdo general acerca de la definición de las empresas de alto crecimiento. Birch y Medoff (1994), por ejemplo, definen a las gacelas como empresas con alto crecimiento de ventas sobre un período. ${ }^{18}$ Otros autores las definen como aquellas que se encuentran en el extremo superior de la distribución de crecimiento de ventas u otra variable (Ahmad 2006). ${ }^{19}$ Recientemente, la OCDE sugiere una definición alternativa para identificar a las empresas de crecimiento acelerado. Específicamente, se definen a las empresas de alto crecimiento como aquellas que crecen por sobre el 20 por ciento por año durante un período mínimo de tres años, con diez o más empleados al inicio del período.

Ante la disparidad de criterios para definir a las empresas de alto crecimiento, en este trabajo se opta por una definición pragmática, que involucra la utilización de las definiciones de tamaño introducidas en la sección anterior. ${ }^{20}$

\section{Definición:}

Se denomina empresas de crecimiento acelerado (gacelas) a aquellas empresas que experimentan transiciones ascendentes en su categoría de tamaño durante una ventana de cinco años.

${ }^{17}$ Henrekson y Johansson (2010) realizan una comprehensiva revisión de la literatura que estudia la contribución de las "gacelas" a la creación de empleo agregado.

${ }^{18}$ Más precisamente, estos autores definen a las gacelas como empresas que experimentan un mínimo de crecimiento de ventas de 20 por ciento por año, con un nivel inicial de ventas de al menos 100 mil dólares (para evitar de esta forma distorsiones originadas por puntos de partida excesivamente bajos).

${ }^{19}$ Una rama diferente de la literatura sugiere agregar a los requisitos anteriores la necesidad de que las empresas en cuestión sean jóvenes, con edad menor a cinco años.

${ }^{20}$ Para corroborar la robustez de los principales resultados, también se utiliza como alternativa la definición de empresa de alto crecimiento de la OCDE en el anexo del trabajo. 
La definición de empresas de crecimiento acelerado implícitamente divide a las unidades productivas, sobrevivientes por períodos de cinco años, en tres grupos distintos. El primero involucra a empresas que no cambian de categoría de tamaño (pequeña, mediana o grande) en la ventana de cinco años. A este tipo de empresas las denominaremos "estacionarias". El segundo grupo de empresas corresponde a las gacelas, las cuales subdividiremos en tres grupos de acuerdo con el tipo de transición de tamaño. El primer tipo de gacela experimenta transiciones de tamaño desde pequeñas a medianas; el segundo tipo pasa de ser mediana a grande; finalmente, el tercer tipo de gacela se transforma de pequeña a grande, siempre sobre la ventana de cinco años. El último grupo de empresas ("resto") corresponde a las que experimentan transiciones descendentes en tamaño (pasan de ser grandes, a medianas o pequeñas, o desde medianas a pequeñas).

Para cuantificar la contribución de las empresas de crecimiento acelerado, el cuadro 5 muestra la contribución de cada tipo de empresa a ventas, empleo y valor agregado. En este cuadro, y en lo que sigue, se utilizan las clasificaciones de tamaño definido en términos de ventas para agrupar a las empresas de crecimiento acelerado y estacionarias. ${ }^{21}$ Las columnas 1 y 2 muestran el número promedio de empresas por tipo para el período 2005-2015. Es importante recalcar que este cuadro sólo considera a las empresas con empleo y ventas positivos al inicio y final de cada ventana de cinco años. Por esta razón, el número total de empresas es considerablemente menor que el número de empresas reportado en el cuadro 4. Como se puede observar, las gacelas son relativamente poco frecuentes. En conjunto, los tres tipos de gacelas representan apenas el 7 por ciento del total de empresas de la economía, correspondiendo la mayor parte de éstas (5 por ciento del total) a empresas que experimentan transiciones desde pequeñas a medianas (gacela 1); seguidas de gacelas que pasan de medianas a grandes (gacela 2), con 1,7 por ciento, y luego empresas que pasan directamente de pequeñas a grandes representan el 0,3 por ciento del total de empresas en la economía.

Las columnas 3 y 4 muestran el empleo agregado explicado por cada categoría en el período t, y al inicio de la ventana de cinco años

${ }^{21}$ Los resultados en que se utilizan clasificaciones de tamaño basadas en empleo son cualitativamente similares, por lo cual se omiten en el resto del artículo. 
Cuadro 5. EMPRESAS DE CRECIMIENTO ACELERADO: CONTRIBUCIÓN A EMPLEO, VENTAS Y VALOR AGREGADO (PROMEDIO 2005-2015)

\begin{tabular}{|c|c|c|c|c|c|c|c|c|}
\hline & (1) & (2) & (3) & (4) & (5) & (6) & (7) & (8) \\
\hline & \multicolumn{2}{|c|}{ Empresas } & \multicolumn{2}{|c|}{ Empleo } & \multicolumn{2}{|c|}{ Ventas } & \multicolumn{2}{|c|}{ Valor agregado } \\
\hline & $\mathrm{N}^{\circ}$ & $\%$ & $t-4$ & $\mathrm{t}$ & $t-4$ & $\mathrm{t}$ & $t-4$ & $\mathrm{t}$ \\
\hline \multicolumn{9}{|l|}{ Estacionarias } \\
\hline Pequeña (estacionaria) & 77.931 & 77,4 & 456 & 470 & 9.595 & 10.612 & 3.813 & 4.155 \\
\hline Mediana (estacionaria) & 8.128 & 8,1 & 319 & 333 & 9.314 & 10.105 & 3.638 & 3.893 \\
\hline Grande (estacionaria) & 5.070 & 5,0 & 1.428 & 1.681 & 95.242 & 119.651 & 32.013 & 37.525 \\
\hline \multicolumn{9}{|l|}{ Crecimiento acelerado } \\
\hline $\begin{array}{l}\text { Gacela } 1 \text { (pequeña a } \\
\text { mediana) }\end{array}$ & 5.084 & 5,0 & 88 & 156 & 2.018 & 4.782 & 799 & 1.871 \\
\hline $\begin{array}{l}\text { Gacela } 2 \text { (mediana a } \\
\text { grande) }\end{array}$ & 1.707 & 1,7 & 104 & 184 & 3.071 & 6.831 & 1.199 & 2.580 \\
\hline $\begin{array}{l}\text { Gacela } 3 \text { (pequeña a } \\
\text { grande) }\end{array}$ & 281 & 0,3 & 6 & 32 & 103 & 1.332 & 33 & 470 \\
\hline Otros & 2.532 & 2,5 & 116 & 53 & 4.468 & 1.382 & 1.457 & 437 \\
\hline Total & 100.732 & 100,0 & 2.517 & 2.909 & 123.811 & 154.696 & 42.953 & 50.931 \\
\hline
\end{tabular}

Nota: El cuadro replica el cuadro 4 utilizando las categorias de tamaño de empresas estacionarias y de crecimiento acelerado que se definen en la sección 4.3. Las categorias de tamaño subyacentes (pequeñas, medianas y grandes) se definen en términos de ventas. Empleo se expresa en miles de trabajadores, mientras que valor agregado y ventas se expresan en miles de millones de pesos de 2013.

que sirve para definir las categorías de tamaño, en t-4. Como se puede observar, las dos primeras categorías de empresas estacionarias (pequeñas y medianas) explican un monto relativamente similar de empleo tanto al inicio como al final de la ventana de cinco años. Comparativamente, las empresas grandes aparecen como mejores agentes creadores de empleo. Cada una de estas empresas añade cerca de 50 puestos de trabajo sobre la ventana de cinco años (unos 250 mil puestos de trabajo). Esto equivale al 55 por ciento del total de los puestos de trabajo creados en el período. ${ }^{22}$

${ }^{22}$ El total del empleo agregado considerado en el cuadro 5 también incluye el empleo de empresas que caen de categoría. Estas empresas se agrupan en la categoría "otros", las cuales contribuyen de forma negativa a la creación total de empleos, "destruyendo" 63 mil empleos en promedio. 
En forma similar a las empresas grandes estacionarias, las gacelas aparecen como agentes extremadamente eficientes en la creación de puestos de trabajo. En conjunto, las gacelas añaden 174 mil puestos de trabajo (25 puestos de trabajo cada empresa), con un equivalente aproximado al 38 por ciento del total de empleos creados en el período. $\mathrm{Si}$ bien este número es menor en términos absolutos que el de las empresas grandes estacionarias, en términos relativos es más significativo. El tamaño promedio de las gacelas al final de la ventana de cinco años es de 50 trabajadores, lo cual implica que la mitad de los puestos de trabajo de estas empresas son creados en la ventana de cinco años. En contraste, los 50 puestos de trabajo creados por las empresas grandes sólo representan el 15 por ciento de los 330 empleados promedio de estas empresas. Al interior de las gacelas, se aprecia una considerable heterogeneidad. El subgrupo de gacelas que pasan de pequeñas a grandes en la ventana de cinco años (gacela 3) es el que experimenta un mayor crecimiento de empleo. Los 26 mil puestos de trabajo creados por estas 281 gacelas implican que cada una de ellas creó en promedio 93 puestos de trabajo; casi el doble que las empresas grandes estacionarias. Estos 93 puestos de trabajos equivalen al 80 por ciento del empleo de este tipo de empresas.

Otra forma de ilustrar la importancia de las gacelas se refleja en el crecimiento de su empleo y ventas en la ventana de cinco años. El crecimiento agregado del empleo en las empresas grandes alcanza 18 por ciento. En contraste el crecimiento promedio en las gacelas es de 88 por ciento, variando desde 78 por ciento en el primer tipo de gacelas, hasta un impresionante 410 por ciento en el tercer tipo de gacela.

Las columnas 5-8 repiten el ejercicio realizado en las columnas 3-4 para las ventas y el valor agregado de las empresas. Las principales conclusiones derivadas para el caso de empleo se mantienen, aunque en términos cuantitativos las diferencias se incrementan. Por ejemplo, el crecimiento de las ventas en el tercer tipo de gacelas crece desde 103 MM de pesos al inicio de la ventana de cinco años, a más de $1.300 \mathrm{MM}$ de pesos al final de la ventana. En contraste, en las empresas grandes las ventas crecen "sólo" 25 por ciento sobre la ventana de cinco años. Las principales conclusiones derivadas hasta este punto se resumen en el siguiente resultado. 


\section{Resultado 2:}

Las empresas de crecimiento acelerado ("gacelas") contribuyen desproporcionadamente a la creación de empleo y valor en la economía en relación con su número y tamaño inicial. Pese a representar sólo el 7 por ciento del total de empresas, en promedio duplican su tamaño en términos de empleo y ventas en una ventana de cinco años, contribuyendo con cerca del 40 por ciento de la creación total de nuevos empleos.

¿Hasta qué punto el resultado anterior depende de la definición dada a las empresas de alto crecimiento? Para responder esta pregunta, el cuadro A1 en el Anexo replica el cuadro 5 utilizando la definición de gacelas de la OCDE (crecimiento superior al 20 por ciento por año durante un período mínimo de tres años, con diez o más empleados al inicio del período). Esta definición podría llevar a encontrar un número distinto de empresas de alto crecimiento por dos razones. Primero, el requisito de crecimiento de ventas es relativamente más exigente, ya que requiere superar un umbral de ventas por un periodo sostenido para ser considerada empresa de alto crecimiento. Por otro lado, la definición OCDE podría llevar a encontrar un mayor número de empresas de alto crecimiento, pues permite identificar como gacelas a empresas que inicialmente ya eran grandes. Al aplicar la definición de gacelas de la OCDE a los datos, se identifican 4.650 gacelas, las cuales representan 4,2 por ciento del total de empresas (ver cuadro A1 en Anexo). Estas empresas añaden 233 mil puestos de trabajos (49,9 puestos de trabajo cada una) sobre una ventana de cuatro años, lo cual equivale a 74,4 por ciento de la creación neta de trabajos. Esto sugiere que, independientemente de la definición aplicada, las empresas de alto crecimiento contribuyen desproporcionadamente al crecimiento de la economía en relación con su tamaño. ${ }^{23}$

${ }^{23}$ Una razón que potencialmente podría explicar la alta contribución de las gacelas al empleo y ventas agregadas sería si éstas estuviesen capturando fusiones y adquisiciones de empresas. Lamentablemente, la información disponible no permite identificar estos episodios. En efecto, si dos empresas se fusionan, esto podría llevar a identificar al conglomerado como una experiencia de alto crecimiento, si es que (i) la fusión siguiera operando con el RUT de una de las empresas fusionadas; y (ii) si ambas empresas, por sí solas, son pequeñas o medianas, y una vez que se fusionan cambian de categoría. Consideramos que este escenario, si bien factible, debería tener baja ocurrencia. 


\section{3. ¿Cuáles son las gacelas?}

En países desarrollados, la mayor parte de las empresas de crecimiento acelerado se encuentran en sectores de alta tecnología. En Chile, en contraste, las gacelas se ubican de manera más uniforme en los distintos sectores productivos. Por ejemplo, en industrias y servicios, la presencia de gacelas se observa en igual proporción a las empresas estacionarias. Las únicas diferencias aparecen en los sectores de la construcción, comercio y transportes: la intensidad de las gacelas es 50 mayor al de empresas estacionarias en construcción y transporte, y 20 por ciento menor en comercio.

En países desarrollados, las gacelas tienen mayor orientación exportadora que las empresas estacionarias, aunque gran parte de ellas comercializan sus productos en el mercado doméstico. Como muestran Bernard et al. $(2003$; 2007) para el caso de Estados Unidos, exportar es una actividad relativamente poco frecuente. En efecto, sólo el 6 por ciento de las empresas chilenas son exportadoras. En contraste, el 12 por ciento de las gacelas son exportadores, aunque al inicio de la ventana de cinco años únicamente el 7 por ciento de las gacelas exportan. Por lo tanto, para sólo una fracción de las gacelas el acceso a los mercados de exportación aparece como una potencial explicación para su rápido crecimiento. ${ }^{24}$

Una pregunta poco explorada en la literatura es si el rápido crecimiento de las gacelas se traduce en mejoras de productividad. Para estudiar esta pregunta, se estiman versiones de la siguiente especificación a nivel de empresas:

$$
y_{i s t}=\alpha_{s t}+\sum_{j \in \text { tamaño }} \delta_{j} D_{i s t}^{j}+\beta \ln \left(\frac{K_{i s t}}{L_{i s t}}\right)+\varepsilon_{i s t}
$$

Donde $y_{i s t}$ denota distintas características de la empresa $i$ en el sector $s$ y período $t, D_{i s t}^{j}$ son variables categóricas que toman el valor uno si la empresa pertenece a cada una de las 7 categorías de tamaño (estacionarias, de crecimiento acelerado y otros; véase nota al pie 23) defini-

${ }^{24}$ Una fracción importante de las gacelas está en sectores no transables. En estos casos, el acceso a los mercados de exportación no es una causa factible para el rápido crecimiento. 
das en el cuadro $5, K_{i s t}$ y $L_{i s t}$ representan el valor libro de los activos fijos y empleo a nivel de empresas, respectivamente, y $a_{i s t}$ denota efectos fijos a nivel de sector-año. Las variables de interés en la ecuación (11) son $\delta_{j}$, las cuales miden la diferencia porcentual en la variable dependiente de cada categoría de tamaño relativo a la categoría de empresas pequeñas estacionarias, que es omitida en la regresión para evitar problemas de colinealidad. Los errores estándares de todas las regresiones son agrupados por sectores. ${ }^{25}$

Los resultados de la estimación de (11) se reportan en el cuadro 6. En las columnas 1 y 2 se muestran los diferenciales en empleo y ventas de cada grupo de empresas. Como se puede apreciar, las gacelas son relativamente más pequeñas que el promedio de empresas del grupo al cual se integran. Por ejemplo, el primer tipo de gacela — que solían ser pequeñas, pero pasan a ser medianas - emplea aproximadamente 25 por ciento menos trabajadores, y tiene ventas 30 por ciento más bajas que el promedio de empresas medianas estacionarias. ${ }^{26}$ Para el caso de las gacelas 2 y 3 - que se integran al grupo de empresas grandes - la desventaja de tamaño con respecto a las empresas grandes estacionarias es aún mayor, llegando a aproximadamente un punto logarítmico para el grupo de empresas que pasaron de pequeñas a grandes en el período de cinco años. ${ }^{27}$

Las columnas 3 y 4 se abocan a documentar los diferenciales de productividad de los distintos tipos de empresas en relación con las empresas pequeñas estacionarias. ${ }^{28}$ Como se puede observar, tanto

${ }^{25}$ La justificación asintótica de estos errores se basa en el número de grupos. Como Cameron et al. (2008) sugieren, un bajo número de grupos - como es nuestro caso- puede redundar en una caída del poder de los contrastes estadísticos. Consecuentemente, en nuestra interpretación de los resultados somos conservadores y evitamos asignar significancia estadística a resultados con significancia al 10 por ciento.

${ }^{26}$ Para obtener estas cifras, basta con restar el coeficiente de las empresas gacelas a las estacionarias. Debido a que ambos coeficientes se expresan relativos a las empresas pequeñas estacionarias, al sustraer ambos coeficientes se cancela la referencia a estas empresas.

${ }^{27}$ A menos que se indique lo contrario, todas las diferencias entre categorías de empresas en el cuadro 6 (y también en el 7) son estadísticamente significativas entre sí al menos al 5 por ciento de significancia. Esto no es sorprendente, considerando el tamaño muestral utilizado en las regresiones.

${ }^{28}$ Los sectores electricidad, gas y agua; administración pública; agropecuario, silvícola y pesquero, y minería son excluidos en las estimaciones, por no contar con información de productividad total de factores. 
Cuadro 6. DIFERENCIALES DE EMPLEO, VENTAS Y PRODUCTIVIDAD EN EMPRESAS DE CRECIMIENTO ACELERADO

\begin{tabular}{|c|c|c|c|c|}
\hline & (1) & (2) & (3) & (4) \\
\hline & \multicolumn{2}{|c|}{ Tamaño } & \multicolumn{2}{|c|}{ Productividad } \\
\hline Variable dependiente & $\log ($ trabaj.) & $\log ($ ventas) & $\log (V A / L)$ & $\log (\mathrm{PTF})$ \\
\hline \multirow[t]{2}{*}{ Mediana (estacionaria) } & $2,409^{* * *}$ & $1,769^{* * *}$ &, $640^{* * *}$ &, $0468^{\star \star *}$ \\
\hline & $(, 0184)$ & $(, 0515)$ & $(, 0440)$ & $(, 00939)$ \\
\hline \multirow[t]{2}{*}{ Grande (estacionaria) } & $4,570^{* * *}$ & $3,290^{* * *}$ & $1,280^{* * *}$ &, $181^{\star * \star}$ \\
\hline & $(, 0283)$ & $(, 0748)$ & $(, 0534)$ & $(, 0144)$ \\
\hline \multirow[t]{2}{*}{ Gacela 1 (pequeña a mediana) } & $2,154^{\star \star *}$ & $1,471^{* \star *}$ &, $683^{* * *}$ &, $0759^{* * *}$ \\
\hline & $(, 0197)$ & $(, 0399)$ & $(, 0295)$ & $(, 00637)$ \\
\hline \multirow[t]{2}{*}{ Gacela 2 (mediana a grande) } & $3,530^{* * *}$ & $2,475^{\star * *}$ & $1,056^{* * *}$ &, $144^{\star * \star}$ \\
\hline & $(, 0238)$ & $(, 0710)$ & $(, 0557)$ & $(, 0119)$ \\
\hline \multirow[t]{2}{*}{ Gacela 3 (pequeña a grande) } & $3,678^{* * *}$ & $2,195^{\star \star *}$ & $1,484^{* * *}$ &, $198^{\star * *}$ \\
\hline & (,0262) & $(, 0447)$ & $(, 0410)$ & $(, 0163)$ \\
\hline \multirow[t]{2}{*}{ Resto } & $1,373^{\star \star *}$ &, $949^{* * *}$ & $.424^{* * *}$ &,$- 0511^{\star * *}$ \\
\hline & $(, 0209)$ & $(, 0292)$ & $(, 0287)$ & $(, 00852)$ \\
\hline EF sector-año & Si & Si & Si & Si \\
\hline R-cuadrado & ,694 &, 528 & , 255 & ,988 \\
\hline Observaciones & 415.333 & 415,333 & 415,333 & 415,333 \\
\hline
\end{tabular}

Nota: El cuadro reporta la diferencia porcentual en la variable dependiente relativa al grupo de empresas pequeñas estacionarias en el periodo 2005-2015. Todas las regresiones son estimadas por mínimos cuadrados ordinarios, y controlan por efectos fijos sector-año y por el logaritmo de la relación capital-trabajo. Errores estándares (en paréntesis) están agrupados a nivel de sectores. Notación: "L" denota trabajadores; "VA" denota valor agregado; "PTF" denota productividad total de los factores. Clave: ${ }^{\star \star \star}$ significativo al 1 por ciento; ${ }^{* \star} 5$ por ciento; ${ }^{*} 10$ por ciento.

en términos de productividad como de productividad total de factores existe una especie de ordenamiento entre productividad y tamaño. Las empresas grandes estacionarias son más productivas que las medianas estacionarias, y éstas, a su vez, más productivas que las pequeñas estacionarias. Lo mismo sucede dentro de las gacelas. Aquellas que transitan a grandes (gacelas 2 y 3 ) son más productivas que las gacelas que pasan de pequeñas a medianas (gacelas 1).

Sorprendentemente, se observa que las gacelas no son significativamente distintas en términos de productividad que la categoría de tamaño a la cual se integran, pese a la desventaja en términos de tama- 
ño. De hecho, las gacelas, en promedio, parecieran ser más productivas que, en promedio también, su nueva categoría de tamaño. Por ejemplo, las gacelas 1 aparecen más eficientes que las empresas medianas estacionarias tanto en términos de productividad laboral como de PTF. Algo similar ocurre con las gacelas 3, las que tienen 20 por ciento más de productividad laboral y 2 por ciento más de PTF que las empresas grandes estacionarias. La única excepción son las gacelas 2, ya que la productividad se encuentra justo entre medio de la productividad de las empresas medianas y grandes estacionarias.

\section{Resultado 3:}

Pese a ser significativamente más pequeñas, al cabo de cinco años las empresas de crecimiento acelerado ("gacelas") cierran prácticamente la totalidad de la brecha de productividad con la categoría de tamaño a la cual se integran.

El hecho de que las gacelas sean relativamente más pequeñas que sus símiles es consistente con dos hipótesis: (i) la escala óptima de las gacelas es menor que la del resto de empresas de su misma categoría de tamaño, y (ii) al final de la ventana de cinco años, las gacelas aún están en transición a su tamaño óptimo, pudiendo éste ser incluso mayor que el del resto de empresas de su grupo. Para entregar una respuesta definitiva sería necesario contar con series de tiempo considerablemente más largas que las utilizadas en este trabajo, buscando determinar cuánto tiempo toma a las empresas alcanzar sus fases de madurez. Sin embargo, bajo el paradigma según el cual el tamaño de las empresas es un reflejo de sus diferencias de productividad, la relativa mayor productividad de las gacelas sugiere que éstas aún no han alcanzado su tamaño óptimo, y que, una vez que alcancen su madurez, ellas debiesen ubicarse en el extremo superior de la distribución de tamaño de las empresas estacionarias.

\subsection{Contribución de las gacelas al crecimiento de la productividad agregada}

No deja de ser sorprendente la similitud en términos de productividad de las gacelas en relación con los grupos de tamaño que integran. ¿Eran las gacelas inicialmente muy productivas, y su rápido crecimien- 
to responde a una transición a su tamaño óptimo? ¿O, por el contrario, en su proceso de rápida expansión las gacelas experimentaron ganancias de eficiencias que les permitieron sostener su alto crecimiento? Dar una respuesta a estas preguntas es de alta relevancia, pues permitirá entender si la contribución de las gacelas al crecimiento de la productividad agregada proviene exclusivamente de reasignación de recursos o si, además, contribuyen al componente de crecimiento al interior de las empresas.

El cuadro 7 muestra los diferenciales de crecimiento sobre una ventana de cinco años para los distintos tipos de empresas. Como se puede apreciar en las columnas 1 y 2, las gacelas experimentan un crecimiento de empleo y ventas significativamente mayores que el grupo de empresas estacionarias. Este resultado no es sorprendente, pues por construcción la expansión de las gacelas requiere de un alto crecimiento de su tamaño. Las columnas 3 y 4 son de mayor interés, donde se reporta el crecimiento de la productividad a través de los distintos tamaños. En relación con las empresas pequeñas, las gacelas son el único grupo que experimenta crecimiento de su PTF. El desempeño de las empresas medianas y grandes estacionarias en términos de crecimiento de la PTF es prácticamente igual al de las empresas pequeñas estacionarias. En contraste, las gacelas experimentan fuertes expansiones de su PTF. Mientras la PTF de los primeros dos tipos de gacela crece entre $5 \mathrm{y}$ 6 por ciento (1,3 y 1,5 por año), la PTF del subgrupo de gacelas que pasan desde pequeñas a grandes crece un impresionante 22 por ciento durante la ventana de cinco años ( 5,1 por ciento por año).

La ventaja competitiva de las gacelas no es particular a la medida de productividad, ni a la definición utilizada para identificar las gacelas. La tercera columna del cuadro 7 muestra que los patrones en términos de productividad laboral son incluso más marcados. Por otro lado, el cuadro A2 (ver Anexo) replica el cuadro 7 utilizando la definición aplicada por la OCDE para identificar a las empresas de alto crecimiento. La ventaja de las gacelas en términos de crecimiento de la PTF llega a casi 12 por ciento por sobre el resto de las empresas, en una ventana de tres años (3,7 por ciento por año). Este valor se encuentra en medio de los valores reportados con la definición base de gacelas utilizada en el cuadro 7. 
Cuadro 7. CRECIMIENTO DE EMPLEO, VENTAS Y PRODUCTIVIDAD EN EMPRESAS DE CRECIMIENTO ACELERADO

\begin{tabular}{|c|c|c|c|c|}
\hline & (1) & (2) & (3) & (4) \\
\hline & \multicolumn{2}{|c|}{ Tamaño } & \multicolumn{2}{|c|}{ Productividad } \\
\hline Variable dependiente & $\Delta^{5}[\log ($ trabaj, $)]$ & $\Delta^{5}[\log ($ ventas $)]$ & $\Delta^{5}[\log (V A / L)]$ & $\Delta^{5}[\log (P T F)]$ \\
\hline \multirow{2}{*}{$\begin{array}{l}\text { Mediana } \\
\text { (estacionaria) }\end{array}$} & ,00363 &, $0616^{* * *}$ &,$- 0580^{* * *}$ &,$- 00905^{\star *}$ \\
\hline & $(, 00456)$ & $(, 00572)$ & $(, 00654)$ & $(, 00349)$ \\
\hline \multirow{2}{*}{$\begin{array}{l}\text { Grande } \\
\text { (estacionaria) }\end{array}$} &, $0938^{* * *}$ &, $136^{* * *}$ &,$- 0423^{* * *}$ &,- 00289 \\
\hline & $(, 00735)$ & $(, 00935)$ & $(, 0102)$ & $(, 00546)$ \\
\hline \multirow{2}{*}{$\begin{array}{l}\text { Gacela } 1 \text { (pequeña a } \\
\text { mediana) }\end{array}$} &, $833^{* * *}$ &, $581^{* \star *}$ & $252^{* * *}$ &, $0525^{* * *}$ \\
\hline & $(, 0152)$ & $(, 0148)$ & $(, 00621)$ & $(, 00441)$ \\
\hline \multirow{2}{*}{$\begin{array}{l}\text { Gacela } 2 \text { (mediana a } \\
\text { grande) }\end{array}$} &, $657^{\star \star *}$ & $.495^{\star \star \star}$ &, $162^{* * *}$ &, $0619^{\star \star *}$ \\
\hline & $(, 0153)$ & $(, 0129)$ & $(, 00752)$ & $(, 00611)$ \\
\hline \multirow{2}{*}{$\begin{array}{l}\text { Gacela } 3 \text { (pequeña a } \\
\text { grande) }\end{array}$} & $2,487^{\star * *}$ & $1,414^{\star \star \star}$ & $1,073^{* * *}$ &, $219^{* * *}$ \\
\hline & $(, 0410)$ & $(, 0435)$ & $(, 0452)$ & $(, 0250)$ \\
\hline \multirow[t]{2}{*}{ Resto } & $-1,128^{\star \star *}$ &,$- 596^{\star \star *}$ &,$- 532^{\star \star *}$ &,$- 114^{* * *}$ \\
\hline & $(, 0251)$ & $(, 0253)$ & $(, 0248)$ & $(, 00865)$ \\
\hline EF sector-año & Si & $\mathrm{Si}$ & $\mathrm{Si}$ & Si \\
\hline R-cuadrado & , 191 & ,113 & 072 & 124 \\
\hline Observaciones & 337,338 & 337,338 & 337,338 & 337,338 \\
\hline
\end{tabular}

Nota: El cuadro reporta la diferencia porcentual en la variable dependiente relativa al grupo de empresas pequeñas estacionarias en el periodo 2005-2015. El operador corresponde a la diferencia en cinco periodos de la variable " $x$ ". Todas las regresiones controlan por efectos fijos sector-año y por el cambio logaritmico (sobre la ventana de cinco años) de la relación capital-trabajo. Errores estándares (en paréntesis) están agrupados a nivel de sectores. Notación: "L" denota trabajadores; "VA" denota valor agregado; "PTF" denota productividad total de los factores. Clave: ${ }^{* * *}$ significativo al 1 por ciento; ${ }^{* \star} 5$ por ciento; ${ }^{*} 10$ por ciento.

Los resultados anteriores sugieren que una gran parte del crecimiento de las empresas sobrevivientes (componente dentro de empresas, o within) se explica por la presencia de gacelas. El crecimiento de la productividad de las gacelas, documentado en el cuadro 7, implica que este grupo, por sí solo, contribuye en 0,75 puntos porcentuales ( 0,2 puntos porcentuales por año) al crecimiento de la productividad agregada en términos de ganancias de eficiencia dentro de las 
empresas. ${ }^{29}$ Esto corresponde a aproximadamente 40 por ciento del crecimiento del componente within de la economía; el restante 60 por ciento es explicado por el conjunto de empresas estacionarias. En contraste a las gacelas, la alta contribución del grupo de empresas estacionarias se explica por su alto peso en la productividad agregada, y no por su dinamismo. En efecto, la PTF en estas empresas sólo crece alrededor de 0,3 por ciento por año, mientras que en las gacelas la productividad crece casi 2,2 por ciento por año. Estos resultados se resumen a continuación

Resultado 4:

Las empresas de crecimiento acelerado contribuyen desproporcionadamente al crecimiento del componente within de la productividad agregada. Pese a contribuir con sólo 8 por ciento de las ventas de la economía, explican cerca del 40 por ciento del crecimiento dentro de las empresas sobrevivientes.

El resultado 4 requiere de tres aclaraciones importantes. Primero, la metodología empleada no permite hacer juicios respecto a la dirección de causalidad del efecto, por lo cual el resultado sólo debe ser interpretado como una descomposición del crecimiento de la productividad. En efecto, tal como lo sugiere la evidencia para Reino Unido (Du y Temouri 2015) y España (Guillamón et al. 2017), pareciera existir un vínculo autorreafirmante entre crecimiento de productividad y crecimiento acelerado: si bien el crecimiento acelerado es seguido de crecimiento de la productividad, un alto crecimiento de la productividad también hace más probable experimentar crecimiento alto en ventas y empleo.

La segunda aclaración, con respecto al resultado 4, se relaciona con el hecho de que el análisis no considera el efecto de la entrada y salida de empresas. Las nuevas empresas suelen ser más eficientes que las que salen (Foster et al. 2008), por lo cual el número reportado sobreestimaría la importancia de las empresas de alto crecimiento sobre la productividad. Sin embargo, la evidencia disponible sugiere una con-

${ }^{29}$ Para llegar a esta cifra, basta con multiplicar la participación de cada tipo de gacelas en las ventas agregadas con el crecimiento de la PTF reportado en la última columna del cuadro 7, agregando el crecimiento promedio de la PTF en empresas pequeñas estacionarias $(0,4$ por ciento al año). 
tribución moderada de la entrada neta de empresas al crecimiento de la productividad agregada, por lo cual el sesgo no debiese cambiar sustantivamente el resultado. ${ }^{30}$

Finalmente, la última aclaración se relaciona con la definición utilizada para definir a las gacelas, pues ésta subestima el número de empresas de crecimiento acelerado inicialmente grandes; de hecho, la definición excluye a gacelas con tamaño inicial grande. Esto podría llevar a subestimar la importancia de las empresas de rápido crecimiento en la productividad agregada. Utilizando la definición de la OCDE para identificar empresas de crecimiento acelerado, la importancia de las gacelas aumenta considerablemente. De acuerdo con esta definición, las gacelas contribuirían 0,7 puntos porcentuales cada año al crecimiento de la PTF en Chile, explicando casi 88 por ciento del crecimiento de la productividad agregada para el período. Por lo tanto, el resultado 4 puede ser considerado como una estimación conservadora de la contribución de las empresas de crecimiento acelerado a la productividad agregada.

\section{5. ¿De dónde proviene la ventaja competitiva de las gacelas?}

Como se explicó anteriormente, la composición sectorial de las gacelas es relativamente similar a la del resto de las empresas, y pese a que tienden a participar más de los mercados de exportación, la mayor parte de las gacelas sólo comercializa sus productos en el mercado doméstico. Por lo tanto, se hace necesario estudiar otras dimensiones que podrían dotar de una ventaja inicial a las gacelas para experimentar un rápido crecimiento.

En este apartado, nos limitamos a realizar un ejercicio simple para determinar la posición inicial de las gacelas en términos de empleo, ventas y productividad.

${ }^{30}$ En horizontes cortos de tiempo, la contribución de la entrada y salida de empresas no supera el 5 por ciento. A medida que el horizonte de tiempo aumenta, la contribución de la entrada neta de empresas tiende a aumentar. En horizontes de cinco años, la contribución a la productividad agregada es aproximadamente un tercio (Melitz y Polanec 2015), mientras que para horizontes a diez años la contribución puede alcanzar aproximadamente 50 por ciento (Bartelsman y Doms 2000). Para más detalles, véase Griliches y Regev (1995) y Baily et al. (2001). 
Debido a que mínimos cuadrados ordinarios es un operador lineal, la situación inicial de la gacela respecto del resto de las empresas puede ser recuperada sustrayendo los coeficientes estimados en el cuadro 7 a aquéllos estimados en el cuadro 6 . El número resultante de esta operación puede interpretarse directamente como el valor de la variable de interés relativo a las empresas pequeñas al inicio de la ventana de cinco años. El resultado de esta operación indica que la productividad inicial de las gacelas es similar al promedio del grupo del cual provienen. Por ejemplo, las gacelas 1 eran inicialmente sólo 2 por ciento más productivas que las empresas pequeñas estacionarias, mientras que las gacelas 2 eran 3 por ciento más productivas que las empresas medianas estacionarias. Las gacelas 3 eran incluso menos productivas (2 por ciento) que el promedio de empresas pequeñas estacionarias. Esto indica que gran parte de la productividad de las gacelas observadas al final de la ventana de cinco años corresponde a ganancias de eficiencia, en términos de reorganización de la producción, incorporación de nuevas tecnologías o mejor uso de los recursos productivos.

La única ventaja de entrada que se observa en las gacelas es en términos de su tamaño inicial. En efecto, los cuadros 6 y 7 sugieren que las gacelas al comienzo eran significativamente más grandes que el grupo de tamaño del cual provenían. En efecto, las gacelas inicialmente pequeñas son 1,2 o 1,3 puntos logarítmicos más grandes en términos de empleo, y entre 0,8 y 0,9 puntos logarítmicos más grandes en términos de ventas que el grupo de empresas pequeñas estacionarias. Las gacelas inicialmente medianas, en tanto, eran 0,46 puntos logarítmicos más grandes en términos de empleo, y 0,2 puntos logarítmicos más grandes en términos de ventas que el grupo de empresas medianas estacionarias.

Las diferencias en tamaño y productividad inicial, si bien son estadísticamente significativas, en términos económicos no parecen ser lo suficientemente radicales como para definirlas como indicadores líderes. Por otro lado, la orientación exportadora de las gacelas no es significativamente distinta del resto de las empresas. Por lo tanto, el mensaje de esta sección sugiere que se requiere más investigación que permita identificar a las gacelas. 


\section{CONCLUSIÓN:}

\section{¿CÓMO AUMENTAR EL CRECIMIENTO DE LA PRODUCTIVIDAD?}

Este trabajo documenta una serie de hechos estilizados respecto a la relación entre tamaño y productividad en la economía chilena. El análisis parte documentando la contribución de los distintos tamaños de empresas a la creación de empleo y valor en la economía chilena. Consistente con evidencia para economías desarrolladas, el trabajo muestra que las empresas grandes son las que explican la mayor parte del empleo, ventas y valor agregado de la economía. Pese a representar una fracción menor del total de empresas, las empresas grandes aportan más de la mitad de los empleos y aproximadamente el 80 por ciento de las ventas de la economía chilena.

Luego, el artículo se enfoca en estudiar la contribución de las empresas de crecimiento acelerado — que denominamos "gacelas" - a la economía. En el trabajo se muestra que las gacelas son el grupo de empresas que aparecen relativamente más dinámicas como agentes creadores de empleo y valor. Pese a representar tan sólo 7 por ciento del total de empresas y 8 por ciento del valor de las ventas, contribuyen con cerca de 40 por ciento de los puestos de trabajos creados sobre una ventana de cinco años. En su conjunto, estos resultados matizan la visión de que las pequeñas empresas son los motores de la economía. Los motores del empleo y el valor de la economía no parecieran ser las empresas pequeñas, sino que las empresas que crecen de manera acelerada, y las empresas que son grandes.

Finalmente, el trabajo muestra que las gacelas son también los agentes más dinámicos en términos de productividad. Pese a representar una pequeña fracción del total de empresas, las gacelas contribuyen con aproximadamente el 40 por ciento del crecimiento de la productividad dentro de empresas. En contraste, el promedio del resto de las empresas pequeñas, medianas y grandes no crece significativamente ni en empleo, valor ni en productividad. Estos resultados sugieren que la productividad agregada podría ser mejorada simplemente fomentando el surgimiento de gacelas. Sin embargo, en la práctica este tipo de empresas sólo pueden ser identificadas ex post facto: su tamaño y productividad iniciales no son significativamente distintos a los del resto de las empresas. Además, se encuentran distribuidas de manera relativamente 
similar al resto de las empresas que componen la economía, y su orientación exportadora tampoco difiere del resto. Esto sugiere que políticas de fomento focalizadas en ciertos tipos de empresas, definidas por tamaño o empleo, puede que no sean exitosas en aumentar la productividad agregada.

Los resultados de este trabajo ponen de manifiesto la necesidad de contar con más investigación que permita entender el surgimiento de gacelas. En particular, ¿por medio de qué mecanismo éstas mejoran su productividad? ¿Se trata simplemente de mejoras organizacionales y de asignación de recursos, o las mejoras en eficiencia involucran mejoras tecnológicas (investigación y desarrollo, incorporación de nuevas tecnologías, etcétera)? ¿Son replicables las gacelas? ¿O, en último término, su surgimiento responde a esfuerzos de individuos o emprendedores particulares, con talentos extraordinarios que difícilmente serán replicables a gran escala? Todas estas son preguntas cuyas respuestas podrán entregar más luces de cómo mejorar la productividad de la economía.

\section{REFERENCIAS}

Ackerberg, D. A., K. Caves \& G. Frazer. 2015. "Structural Identification of Production Functions". Econometrica 83 (6): 2411-2451.

Ahmad, N. 2006. "A Proposed Framework for Business Demographic Statistics". OECD Statistics Working Paper Series, STD/DOC, 3, París.

Asker, J., A. Collard-Wexler \& J. De Loecker. 2014. "Dynamic Inputs and Resource (Mis) Allocation". Journal of Political Economy 122 (5): 1013-1063.

Baily, Martin N., Eric J. Bartelsman \& John Haltiwanger. 2001. "Labor Productivity: Structural Change and Cyclical Dynamics". The Review of Economics and Statistics 83 (3): 420-433.

Bartelsman, E. J. \& M. Doms. 2000. "Understanding Productivity: Lessons from Longitudinal Microdata”. Journal of Economic Literature 38 (3): 569-594.

Bernard, A. B., J. Eaton, J. B. Jensen \& S. Kortum. 2003. "Plants and Productivity in International Trade". American Economic Review 93 (4): 1268-1290.

Bernard, A. B., J. B. Jensen, S. J. Redding \& P. K. Schott. 2007. "Firms in International Trade". Journal of Economic Perspectives 21 (3): 105-130.

Bianchini, S., G. Bottazzi \& F. Tamagni. 2017. "What does (not) characterize persistent corporate high-growth?” Small Business Economics 48 (3): 633656.

Birch, D. L. 1979. The Job Generation Process. MIT Program on Neighborhood and Regional Change, Massachusetts Institute of Technology, Cambridge, MA. 
1981. "Who Creates Jobs?". The Public Interest 65: 3-14.

1987. Job Creation in America. New York: Free Press.

Birch, D. L. \& J. Medoff. 1994. “Gazelles”. En Labor Markets, Employment Policy and Job Creation, editado por Lewis C. Solmon \& Alec R. Levenson, 159168. Boulder: Westview Press.

Canales, M. \& A. García Marín. 2018. "Productividad, tamaño y empresas súperestrella: Evidencia microeconómica para Chile”. Documento de Trabajo 458, Universidad de Chile.

Cameron, A. C., J. B. Gelbach \& D. L. Miller. 2008. "Bootstrap-Based Improvements for Inference with Clustered Errors". The Review of Economics and Statistics 90: 414-427.

Comisión Nacional de Productividad. 2017. Informe Anual 2017. Comisión Nacional de Productividad, Santiago.

Criscuolo, C., P. Gal \& C. Menon. 2014. "The Dynamics of Employment Growth: New Evidence from 18 Countries". OECD Science, Technology and Industry Policy Papers 14. OECD Publishing, París. http://dx.doi. org/10.1787/5jz417hj6hg6-en

Daunfeldt, S-O. \& D. Halvarsson. 2015. “Are High-Growth Firms One-Hit Wonders? Evidence from Sweden”. Small Business Economics 44 (2): 361-383.

Davis, S. J., J. Haltiwanger \& S. Schuh. 1996a. Job Creation and Destruction. Cambridge, MA: MIT Press.

- 1996b. "Small Business and Job Creation: Dissecting the Myth and Reassessing the Facts”. Small Business Economics 8 (4): 297-315.

De Loecker, J. 2011. "Product Differentiation, Multiproduct Firms, and Estimating the Impact of Trade Liberalization on Productivity". Econometrica 79 (5): 1407-1451.

Du, J. \& Y. Temouri. 2015. "High-Growth Firms and Productivity: Evidence from the United Kingdom”. Small Business Economics 44 (1): 123-143.

Eslava, M. \& J. C. Haltiwanger. 2018. "The Life-Cycle Growth of Plants: The Role of Productivity, Demand and Distortions". Mimeo. http://dx.doi.org/10.2139/ ssrn.3177289.

Foster, L., J. Haltiwanger \& C. Syverson. 2008. "Reallocation, Firm Turnover, and Efficiency: Selection on Productivity or Profitability?" American Economic Review 98 (1): 394-425.

- 2016. "The Slow Growth of New Plants: Learning about Demand?". Economica 83 (329): 91-129.

García Marín, A. \& N. Voigtländer. (Por aparecer). "Exporting and Plant-Level Efficiency Gains: It's in the Measure". Journal of Political Economy.

Goswami, A. G., D. Medvedev \& E. Olafsen. 2018. High-Growing Firms: Facts, Fiction, and Policy Options for Emerging Economies. Washington, D.C.: World Bank Group.

Griliches, Z. \& H. Regev. 1995. "Firm productivity in Israeli industry 1979-1988". Journal of Econometrics 65 (1): 175-203. 
Guillamón, C., E. Moral-Benito \& S. Puente. 2017. "High Growth Firms in Employment and Productivity: Dynamic Interactions and the Role of Financial Constraints". Banco de España Working Paper 1718. http://dx.doi. org/10.2139/ssrn.2967223.

Hall, R. E. \& C. I. Jones. 1999. "Why do Some Countries Produce So Much More Output Per Worker than Others?" The Quarterly Journal of Economics 114 (1): 83-116.

Haltiwanger, J., R. S. Jarmin \& J. Miranda. 2013. "Who Creates Jobs? Small Versus Large Versus Young”. The Review of Economics and Statistics 95 (2): 347361.

Henrekson, M. \& D. Johansson. 2010. "Gazelles as Job Creators: A Survey and Interpretation of the Evidence". Small Business Economics 35: 227-244.

Hsieh, C. T. \& P. J. Klenow. 2009. "Misallocation and Manufacturing PTF in China and India". Quarterly Journal of Economics 124 (4): 1403-1448.

. 2014. "The Life Cycle of Plants in India and Mexico". Quarterly Journal of Economics 129 (3): 1035-1084.

Klette T. J. \& Z. Griliches. 1996. "The Inconsistency of Common Scale Estimators When Output Prices Are Unobserved and Endogenous". Journal of Applied Econometrics 11: 343-361.

Levinsohn, J. \& A. Petrin. 2003. "Estimating Production Functions Using Inputs to Control for Unobservables". Review of Economic Studies 70 (2): 317-341.

Levinsohn, J. \& M. Melitz. 2006. "Productivity in a Differentiated Products Market Equilibrium". Mimeo.

Lucas, R. E. 1978. "On the Size Distribution of Business Firms". The Bell Journal of Economics 9 (2): 508-523.

Melitz, M. \& S. Polanec. 2015. "Dynamic Olley-Pakes Productivity Decomposition with Entry and Exit". RAND Journal of Economics 46 (2): 362-375.

Midrigan, V. \& D.Y. Xu. 2014. "Finance and Misallocation: Evidence from PlantLevel Data". American Economic Review 104 (2): 422-458.

OECD. 2018. "Financing SMEs and Entrepreneurs 2018: An OECD Scoreboard". http://www.oecd.org/cfe/smes/financing-smes-and-entrepreneurs-23065265. $\mathrm{htm} /$.

Olley, G. S. \& A. Pakes. 1996. "The Dynamics of Productivity in the Telecommunications Equipment Industry”. Econometrica 64 (6): 1263-1297.

Restuccia, D. \& R. Rogerson. 2013. "Misallocation and Productivity". Review of Economic Dynamics 16 (1): 1-10.

- 2017. "The Causes and Costs of Misallocation". Journal of Economic Perspectives 31 (3): 151-174. 


\section{ANEXO. CUADROS ADICIONALES}

Cuadro A1. EMPRESAS DE CRECIMIENTO ACELERADO, SEGÚN DEFINICIÓN OCDE: CONTRIBUCIÓN A EMPLEO, VENTAS Y VALOR AGREGADO. PROMEDIO 2005-2015.

\begin{tabular}{lcccccccc}
\hline & $(1)$ & $(2)$ & $(3)$ & $(4)$ & $(5)$ & $(6)$ & $(7)$ & $(8)$ \\
\hline & \multicolumn{2}{c}{ Empresas } & \multicolumn{2}{c}{ Empleo } & \multicolumn{2}{c}{ Ventas } & \multicolumn{2}{c}{ Valor agregado } \\
\hline & $\mathrm{N}$ & $\%$ & $\mathrm{t}-3$ & $\mathrm{t}$ & $\mathrm{t}-3$ & $\mathrm{t}$ & $\mathrm{t}-3$ & $\mathrm{t}$ \\
\hline $\begin{array}{l}\text { Crecimiento } \\
\text { acelerado }\end{array}$ & 4.650 & 4,2 & 305 & 537 & 11.706 & 29.647 & 3.977 & 9.510 \\
OCDE & & & & & & & & \\
Resto & 106.590 & 95,8 & 2.406 & 2.486 & 121.683 & 128.271 & 41.682 & 42.787 \\
\hline Total & 111.240 & 100 & 2.711 & 3.023 & 133.389 & 157.918 & 45.659 & 52.297 \\
\hline
\end{tabular}

Nota: El cuadro muestra la contribución de las distintas categorias de tamaño de empresas estacionarias y de crecimiento acelerado definidas de acuerdo a la OCDE en términos de número (columnas 1 y 2), nivel y crecimiento de empleo (columnas 3 y 4), ventas (columnas 5 y 6) y valor agregado (columnas 7 y 8). La OCDE define empresas de crecimiento acelerado como aquellas con crecimiento promedio de ventas superior al 20 por ciento anual sobre un periodo de tres años, con un nivel de empleo inicial de al menos diez trabajadores, con al menos cuatro años presente en la muestra. Empleo se expresa en miles de trabajadores, mientras que valor agregado y ventas se expresan en miles de millones de pesos de 2013.

Cuadro A2. CRECIMIENTO DE EMPLEO, VENTAS Y PRODUCTIVIDAD EN EMPRESAS DE CRECIMIENTO ACELERADO DE ACUERDO A LA DEFINICIÓN OCDE.

\begin{tabular}{lrrrr}
\hline & \multicolumn{1}{c}{ (1) } & (2) & (3) & (4) \\
\hline Variable dependiente & \multicolumn{2}{c}{ Tamaño } & \multicolumn{2}{c}{ Productividad } \\
\hline Crecimiento acelerado OCDE & log(ventas) & \multicolumn{1}{c}{$\log (\mathrm{L})$} & $\log (\mathrm{VA} / \mathrm{L})$ & $\log (\mathrm{PTF})$ \\
\hline Efecto fijo sector-año &, $861^{* * *}$ &, $424^{* * *}$ &, $426^{* * *}$ &, $119^{* * *}$ \\
R-cuadrado & $(, 0139)$ & $(, 0106)$ & $(, 0087)$ & $(, 00565)$ \\
Observaciones & $\mathrm{Si}$ & $\mathrm{Si}$ & $\mathrm{Si}$ & $\mathrm{Si}$ \\
\hline
\end{tabular}

Nota: El cuadro reporta la diferencia porcentual en la variable dependiente entre las empresas de crecimiento acelerado de acuerdo a la definición de la OCDE en relación con el resto de empresas en el periodo 2005-2015. Todas las regresiones controlan por efectos fijos sector-año y por el logaritmo de la relación capital-trabajo, definida al nivel de empresa-año. Errores estándares (en paréntesis) están agrupados a nivel de sectores. Notación: "L" denota trabajadores; "VA" denota valor agregado; "PTF" denota productividad total de los factores. Clave: ${ }^{* * *}$ significativo al 1 por ciento; ${ }^{* *} 5$ por ciento; * 10 por ciento. 